\title{
Efficacy of trastuzumab plus paclitaxel for HER2-positive gastroesophageal junction cancer that is refractory to $\mathrm{S}-1$
}

\author{
Setsuo Utsunomiya $\cdot$ Takuya Kurimoto
}

Received: 10 September 2012/ Accepted: 15 November 2012/Published online: 20 December 2012

(C) The Japan Society of Clinical Oncology 2012

\begin{abstract}
A 60-year-old woman was seen in our hospital because of dysphagia and upper abdominal pain. Endoscopic examination revealed advanced gastroesophageal junction (GEJ) cancer, and computed tomography (CT) scan of the abdomen revealed multiple liver and lymph node metastases. Chemotherapy with S-1 plus cisplatin was administered, but the patient did not tolerate this chemotherapy because of the side effects. Although chemotherapy with S-1 alone was administered, CT scan after 1 month of treatment showed tumor progression. Since the human epidermal growth factor receptor 2 (HER2) status of her GEJ cancer specimens was strongly positive, trastuzumab plus paclitaxel was administered. One month later, CT scan showed marked tumor shrinkage. Grade 2 alopecia and grade 1 peripheral neuropathy developed. In this case, chemotherapy with trastuzumab plus paclitaxel was effective and safe for HER2-positive GEJ cancer that was refractory to $\mathrm{S}-1$.
\end{abstract}

Keywords Gastric cancer · HER2 - Trastuzumab · Paclitaxel

S. Utsunomiya $(\bowtie)$

Department of Clinical Oncology, Kainan Hospital,

396 Minamihonden, Maegasu-cho, Yatomi, Aichi 498-8502,

Japan

e-mail: utsunomiya162@gmail.com

T. Kurimoto

Department of Gastrointestinal Oncology, Nagoya Kyoritsu

Hospital, Nagoya, Japan

\section{Introduction}

Trastuzumab is a monoclonal antibody against human epidermal growth factor receptor 2 (HER2) and has shown survival benefits when given with chemotherapy in patients with HER2-positive breast cancer. In a randomized phase III trial (the ToGA trial), trastuzumab in combination with standard chemotherapy (capecitabine plus cisplatin or fluorouracil plus cisplatin) showed survival benefits in patients with HER2-positive gastric or gastroesophageal junction (GEJ) cancer [1]. However, the efficacy and safety of trastuzumab in combination with any other cytotoxic agents for HER2-positive gastric or GEJ cancer have not been evaluated. Here, we report a case of advanced GEJ cancer that responded to chemotherapy with trastuzumab plus paclitaxel after progression on chemotherapy with S-1.

\section{Case report}

In January 2011, a 60-year-old woman was seen in our hospital because of dysphagia and upper abdominal pain. Esophagogastroduodenoscopy (EGD) revealed an ulcerated mass in the cardia of the stomach (Fig. 1). The biopsy specimens were positive for adenocarcinoma (Fig. 2a). Computed tomography (CT) scan of the abdomen revealed multiple liver and lymph node metastases (Fig. 3a, b). The level of hemoglobin was $11.5 \mathrm{~g} / \mathrm{dl}$ (reference range $12-16 \mathrm{~g} / \mathrm{dl}$ ), and cancer antigen 19-9 (CA19-9) was $2014 \mathrm{U} / \mathrm{ml}$ (reference range $<37 \mathrm{U} / \mathrm{ml}$ ). Other laboratory tests were normal, including carcinoembryonic antigen (CEA), electrolytes, hepatic and renal function.

Chemotherapy with S-1 plus cisplatin was initiated. S-1 is an orally active combination of tegafur (a prodrug that is converted to fluorouracil), gimeracil (an inhibitor of 


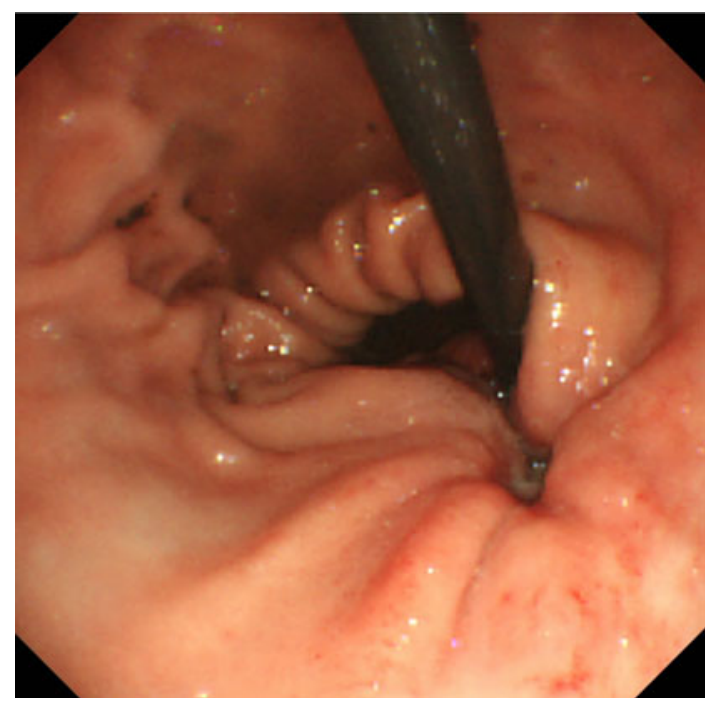

Fig. 1 Esophagogastroduodenoscopy (EGD) acquired before treatment. EGD revealed a large ulcerated mass in the cardia of the stomach extending to the gastroesophageal junction

dihydropyrimidine dehydrogenase, which degrades fluorouracil) and oteracil (which inhibits the phosphorylation of fluorouracil in the gastrointestinal tract, thereby reducing the gastrointestinal toxic effects of fluorouracil). She improved clinically with resolution of dysphagia and abdominal pain. CT scan demonstrated that liver and lymph node metastases had reduced in size. Although chemotherapy was to be effective for her disease, grade 3 febrile neutropenia and anorexia developed after two cycles of chemotherapy. She declined chemotherapy with S-1 plus cisplatin, after recovering from the side effects. Therefore, we treated her with S-1 alone. However, her symptoms had become worse, and liver and lymph node metastases enlarged (Fig. 3c, d).

The HER2 status of her gastroesophageal junction (GEJ) cancer specimens was positive as evaluated by

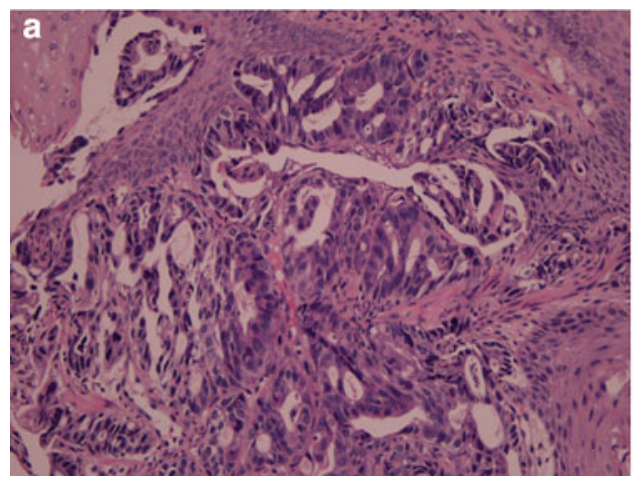

Fig. 2 Pathological specimens of the gastroesophageal junction cancer. a Biopsy specimens showed moderately differentiated tubular adenocarcinoma. b Human epidermal growth factor receptor 2 status was evaluated by immunohistochemistry (HercepTest, DAKO, immunohistochemistry (HercepTest ${ }^{\mathrm{TM}}$, DAKO, Copenhagen, Denmark, Fig. 2b) and by fluorescence in situ hybridization (FISH; HER2 FISH pharmDx, DAKO). Echocardiography showed normal ventricular size and function. She would like to receive further chemotherapy with mild toxicity. In July 2011, trastuzumab plus paclitaxel was initiated. Trastuzumab was administered at $8 \mathrm{mg} /$ $\mathrm{kg}$ as loading dose followed by $6 \mathrm{mg} / \mathrm{kg}$ every 3 weeks. Paclitaxel was administered at $80 \mathrm{mg} / \mathrm{m}^{2}$ weekly, three times every 4 weeks. Her symptoms of dysphagia and abdominal pain improved immediately. One month after initiation of this treatment, liver and lymph node metastases had markedly reduced in size (Fig. 3e, f). The level of CA 19-9 was decreased from more than 12000 to 4179 U/ $\mathrm{ml}$. Grade 2 alopecia and grade 1 peripheral neuropathy developed. Two months later, her symptoms had become worse, and CT scan showed considerable disease progression. Although chemotherapy with irinotecan was administered, her disease did not respond to the treatment. She has received best supportive care since we decided to discontinue chemotherapy in November 2011.

\section{Discussion}

A randomized phase III trial (the ToGA study) compared the efficacy and safety of trastuzumab plus standard chemotherapy (capecitabine plus cisplatin or fluorouracil plus cisplatin) with those of standard chemotherapy alone for patients with advanced gastric or GEJ cancer that overexpressed HER2. Trastuzumab plus chemotherapy resulted in a significant improvement of response rate (47.3 vs. $34.5 \%$, $p=0.0017$ ), progression-free survival (6.7 vs. 5.5 months, $p=0.0002)$ and overall survival (13.8 vs. 11.1 months; hazard ratio $0.74, p=0.0046$ ) [1]. However, the efficacy and safety of trastuzumab plus any other cytotoxic agents for advanced gastric or GEJ cancer have not been evaluated.

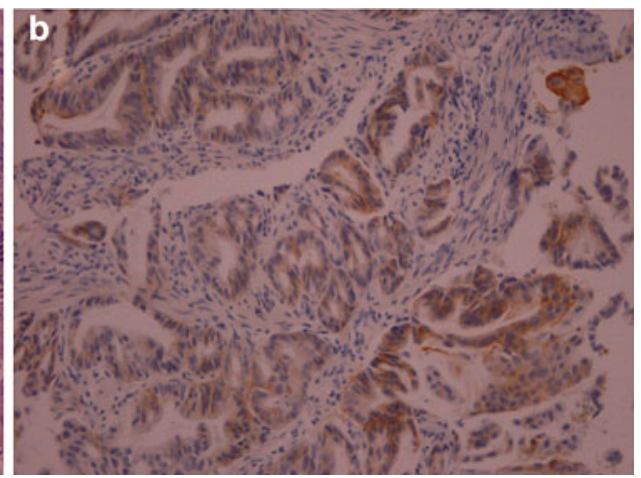

Copenhagen, Denmark) and was found to be positive (2+). It was also evaluated by fluorescence in situ hybridization (HER2 FISH pharmDx, DAKO) and was positive (HER2/CEP17 ratio = 8.03) 


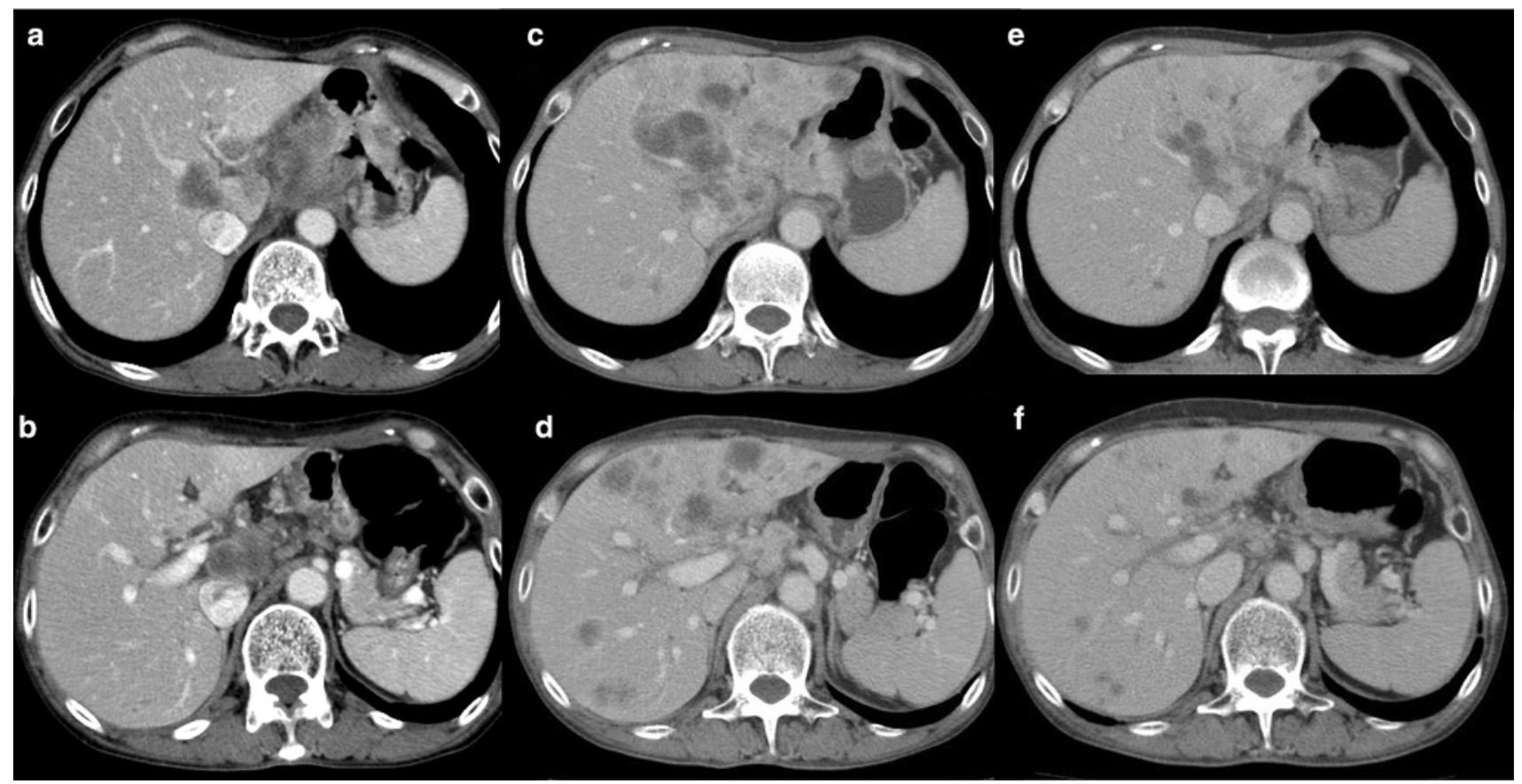

Fig. 3 Computed tomography (CT) scan acquired before S-1 plus cisplatin, and before and after trastuzumab plus paclitaxel. a, b CT scan acquired before treatment with $\mathrm{S}-1$ plus cisplatin revealed multiple liver and lymph node metastases. c, d CT scan acquired before treatment with trastuzumab plus paclitaxel showed multiple,

On the other hand, in a randomized phase III trial, the addition of trastuzumab to chemotherapy (doxorubicin plus cyclophosphamide, epirubicin plus cyclophosphamide, or paclitaxel) for patients with metastatic breast cancer that overexpressed HER2 resulted in a significant improvement of time to disease progression (7.4 vs. 4.6 months; $p<0.001)$, rate of objective response (50 vs. $32 \%$; $p<0.001)$ and survival (25.1 vs. 20.3 months; $p=0.046$ ) [2]. In the preclinical study, trastuzumab plus cytotoxic agents (capecitabine, cisplatin, irinotecan, docetaxel and pacelitaxel) showed more potent antitumor activity than the cytotoxic agents alone in HER2-overexpressing human gastric cancer xenograft models [3].

S-1 refractory gastric cancer may not respond to treatment of capecitabine, because both $\mathrm{S}-1$ and capecitabine are a prodrug of 5-fluorouracil. Thus, capecitabine-based chemotherapy is not considered an appropriate treatment for our patient with GEJ cancer refractory to S-1. A single study evaluated the feasibility of trastuzumab plus irinotecan in ten patients with HER2-positive metastatic breast cancer (MBC). In this study, grade 3 or 4 neutropenia occurred in three patients, and one of them died of treatment-related sepsis [4].

At present, trastuzumab in combination with taxanes (paclitaxel administered weekly or docetaxel every 3 weeks) is the standard of care for patients with HER2positive MBC. Phase II studies evaluated the efficacy and large metastatic lesions in the liver and lymph nodes. e, f CT scan acquired 1 month after the initiation of trastuzumab plus paclitaxel. Multiple liver and lymph node metastases appeared to be markedly reduced in size

safety of trastuzumab plus taxanes for HER2-positive MBC. Trastuzumab in combination with paclitaxel or docetaxel had similar efficacy in the treatment of MBC, but different toxicity profiles. In these studies, grade 3 or 4 neutropenia and febrile neutropenia were seen more commonly with trastuzumab plus docetaxel (32 and $23 \%$ ) than with trastuzumab plus weekly paclitaxel (6 and $2 \%)[5,6]$.

Therefore, we consider trastuzumab plus paclitaxel for our patient with GEJ cancer that overexpressed HER2. In summary, trastuzumab plus paclitaxel resulted in marked and rapid tumor shrinkage. Severe adverse events (Grade 3 or 4) due to this regimen were not seen. However, the duration of tumor response was shorter than 2 months.

Further prospective study of this combination chemotherapy for HER2-positive gastric or GEJ cancer that is refractory to $\mathrm{S}-1$ is warranted, because many patients with gastric or GEJ cancer receive adjuvant chemotherapy with S-1 after curative resection in Japan.

Conflict of interest The authors declare that they have no conflict of interest.

\section{References}

1. Bang YJ, Van Cutsem E, Feyereislova A et al (2010) Trastuzumab in combination with chemotherapy versus chemotherapy alone for treatment of HER2-positive advanced gastric or gastro-oesophageal 
junction cancer (ToGA): a phase 3, open-label, randomized controlled trial. Lancet 28:687-697

2. Slamon DJ, Leyland-Jones B, Shak S et al (2001) Use of chemotherapy plus a monoclonal antibody against HER2 for metastatic breast cancer that overexpresses HER2. N Engl J Med 15:783-792

3. Fujimoto-Ouchi K, Sekiguchi F, Yasuno H et al (2007) Antitumor activity of trastuzumab in combination with chemotherapy in human gastric cancer xenograft models. Cancer Chemother Phamacol 59:795-805

4. Ikeda M, Kurebayashi J, Sonoo H et al (2009) Evaluation of irinotecan hydrochloride (CPT-11) and trastuzumab combination therapy as salvage treatment in patients with HER2 overexpressing metastatic breast cancer. Jpn J Cancer Chemother 36:773-777

5. Seidman AD, Fornier MN, Esteva FJ et al (2001) Weekly trastuzumab and paclitaxel therapy for metastatic breast cancer with analysis of efficacy by HER2 immunophenotype and gene amplification. J Clin Oncol 19:2587-2595

6. Marty M, Cognetti F, Maraninchi D et al (2005) Randomized phase II trial of the efficacy and safety of trastuzumab combined with docetaxel in patients with human epidermal growth factor receptor 2-positive metastatic breast cancer administered as firstline treatment: the M77001 Study Group. J Clin Oncol $23: 4265-4274$ 